\title{
Efficient virotherapy of cancer in the presence of immune response
}

\author{
Binayak S. Choudhury • Bankim Nasipuri
}

Received: 1 August 2013 / Revised: 28 October 2013 / Accepted: 30 October 2013 / Published online: 15 November 2013

(C) Springer-Verlag Berlin Heidelberg 2013

\begin{abstract}
In recent times the possibilities of treating cancer using specific types of viruses are being explored. Here we consider a nonlinear mathematical model based on ordinary differential equations to study the efficacy of cancer therapy using oncolytic viruses in the presence of immune response. A cancer-immune model that describes complex interactions underlying the ability of the immune system to inhibit tumor growth along with its effects on the spread of oncolytic virus population is studied. By using numerical simulations we analyze the influence of parameters of the model on the outcome of the therapy. Our finding is that it is possible to control the cancer, even sometimes to extinct it, if we can properly control some parameters relating either to the body system or to the administration of the virotherapy.
\end{abstract}

Keywords Tumor · Oncolytic virus · Virotherapy · Immune response $\cdot$ Mathematical model

\section{Introduction}

In search of effective therapies of cancer, the possible use of viruses has invoked interest in recent years. This branch of study is known as tumor virotherapy. Some viruses like Newcastle disease virus, Vesicular stomatities virus, etc. are examples of viruses that infect the tumor cells. There are

\footnotetext{
B. S. Choudhury · B. Nasipuri

Department of Mathematics, Bengal Engineering and Science

University, Shibpur P.O. : B. Garden, Shibpur, Howrah 711103,

West Bengal, India

e-mail: binayak12@yahoo.co.in

B. Nasipuri $(\bowtie)$

Department of Mathematics, Prabhu Jagatbandhu College, University of Calcutta, P.O. : Andul-Mouri, Howrah 711302, West Bengal, India e-mail: bnasipuri@gmail.com
}

also viruses like ONYX-015 (Adenovirus) which are artificially engineered for this specific purpose. The aim of this therapy is to infect the tumor cells with these viruses which will be extinct together the tumor cells while leaving the nontumor cells more or less unaffected. Some important recent references on this topic are [1-18] etc. and the references therein. At the same time the role of body immune system in the context of the progress of cancer cells has been discussed in a number of works. An early reference is due to Burnet [19] in which it was hypothesized that the progression of cancer is possible only when the immune response system remains nonreactive to certain proteins synthesized by the cancer cells. The phenomena is technically known as tolerance. In general, immune responses are largely of two types, one is the innate immune response and the other is adaptive immune response. It is the later which is the active part of the immunological system. Again, broadly they are of two types, the antibodies and the killer cells. While the antibodies fight the foreign elements like viruses, bacteria etc. by recognizing foreign proteins outside the cells, the killer cells recognize the mutated proteins of the cancer cells on display on cell walls. These killer cells are also known as cytotoxic $\mathrm{T}$ lymphocytes (CTL). After the recognition, the CTLs undergo a process of cell division. Then they release certain molecules, perforin for instance, which kills the cancer affected cells. The process is called reactivity. It is also possible that the CTLs fail to recognize the mutated proteins as different from normal proteins of the body. In this case the phenomena of tolerance is said to take place. It is believed that the progress of cancer is in the period when the adaptive immune system exhibits tolerance. Although, in the recent times, we do not have full understanding of the immunological principles related to the problem of cancer, it is certain, as suggested by recent studies, that such relations are present in some form. In this paper we present a mathematical model where we con- 
sider the efficiency of virotherapy in the presence of immune responses of the body. Our approach is through numerical simulations. It is revealed that if it is possible to adjust values of certain parameters, either associated with the body system or with the administering virus, then the virotherapy can be effective in the treatment of cancer.

The remaining portion of this paper is organized as follows: In Sect. 2, we briefly discuss positivity and boundedness of the mathematical model, examine the conditions for the existence of positive equilibrium states and stability criteria of infection-free and interior equilibria. Next we demonstrate the occurrence of backward bifurcation phenomena. Section 3 deals with numerical illustrations showing typical dynamical behaviours of our basic model. Lastly we give some remarks in concluding Sect. 4.

\section{Mathematical model}

We consider here the following system of non-linear ordinary differential equations that models the complex dynamics of the population of two types of tumor cells, uninfected and infected, and immune response over time $t$

$$
\begin{aligned}
& \frac{d x}{d t}=\phi(x, y) x-\beta \psi(x, y) y, \\
& \frac{d y}{d t}=\beta \psi(x, y) y-\delta y-p y z, \\
& \frac{d z}{d t}=f(y, z)-q z,
\end{aligned}
$$

where $x=x(t)$ stands for the uninfected tumor cell population, $y=y(t)$ the infected tumor cell population and $z=z(t)$ the population of the virus specific CTLs. The function $\phi(x, y)$ describes the growth and death processes of the uninfected tumor cells and the function $\psi(x, y)$ describes the rate at which the tumor cells become infected by the virus. These two functions can take several different forms depending on how much detail of the biology is incorporated into the model. The coefficient $\beta$ represents the infectivity or the transmission rate which also includes the replication rate of viruses. The deaths of virus infected cells occur at a rate $\delta y, \delta$ is called the viral cytotoxicity. The function $f(y, z)$ describes the rate of immune response due to virus activation. In the absence of antigenic stimulation the CTLs die with a rate $q z$. The particular form of this response depends on different assumptions [20-22]. Here we assume that the production of CTLs depends on both the population of infected cells and CTL cells, for this reason we assume that $f(y, z)=\gamma y z$, where $\gamma$ stands for the strength of the CTL response or CTL responsiveness [12]. Infected cells are destroyed by the CTL response at a rate $p y z$, corresponding to lytic effector mechanisms of CTL response, where the coefficient $p(>0)$ represents the strength of the lytic component $[23,24]$. The logis- tic growth is a widely used growth pattern, here we assume tumor grows logistically with maximum size the tumor is allowed to occupy is given by its carrying capacity $k$. We, therefore, take $\phi(x, y)$ in the form

$\phi(x, y)=r\left(1-\frac{x+y}{k}\right)$,

where $r$ is the maximum per capita growth rate of uninfected tumor cells. We consider the virus spread term $\psi(x, y)$ in the form $\frac{x}{x+y+\alpha}$, a fast virus spreading term. The rationale behind the assumption of the fast virus spreading term is discussed in detail in [5]. Based on these assumptions the model (1a, $1 \mathrm{~b}, 1 \mathrm{c})$ takes the following form

$$
\begin{aligned}
& \frac{d x}{d t}=r x\left(1-\frac{x+y}{k}\right)-\beta \frac{x y}{x+y+\alpha}, \\
& \frac{d y}{d t}=\beta \frac{x y}{x+y+\alpha}-\delta y-p y z, \\
& \frac{d z}{d t}=\gamma y z-q z .
\end{aligned}
$$

All the parameters of the system are assumed to be positive with initial populations $x(0) \geq 0, y(0) \geq 0, z(0) \geq 0$.

\subsection{Positivity and boundedness of the solutions}

For the well possness of the biological system, we first check the positivity and boundedness of the system $(2 \mathrm{a}-2 \mathrm{c})$.

Theorem 2.1.1 All solutions of the system (2a-2c) are positive and bounded subject to the given initial conditions $x(0) \geq 0, y(0) \geq 0, z(0) \geq 0$.

Proof Equation (2a) can be written as $\frac{d x}{x}=\psi_{1}(x, y) d t$, where $\psi_{1}(x, y)=r\left(1-\frac{x+y}{k}\right)-\beta \frac{y}{x+y+\alpha}$.

Integrating over $[0, \mathrm{t}]$, we can obtain $x(t)=x(0)$ $e^{\int_{0}^{t} \psi_{1}(x, y) d t} \geq 0$ for all $t \geq 0$ as $x(0) \geq 0$.

Again equation (2b) gives $\frac{d y}{y}=\psi_{2}(x, y, z) d t$,

where $\psi_{2}(x, y, z)=\beta \frac{x}{x+y+\alpha}-\delta-p z$.

By integration in the range $[0, \mathrm{t}]$, we again see that $y(t)=$ $y(0) e^{\int_{0}^{t} \psi_{2}(x, y, z) d t} \geq 0$ for all $t \geq 0$ as $y(0) \geq 0$. Similarly from (3c), $z(t) \geq 0$ for all $t \geq 0$.

To show all solutions will remain bounded we proceed as follows. From (2a), we have $\frac{d x}{d t} \leq r x\left(1-\frac{x+y}{k}\right) \leq$ $r x\left(1-\frac{x}{k}\right)$, which gives $\lim \sup _{t \rightarrow \infty} x(t) \leq k$, by usual comparison theorem. Again we notice that

$$
\begin{aligned}
\frac{d}{d t}(x+y) & =r x\left(1-\frac{x+y}{k}\right)-\delta y-p y z \\
& \leq r x\left(1-\frac{x+y}{k}\right) \leq r k\left(1-\frac{x+y}{k}\right),
\end{aligned}
$$

which also gives $\lim \sup _{t \rightarrow \infty}(x(t)+y(t)) \leq k$ for all $t \geq 0$. 
Now, To find the bound of $z$, we consider $V(t)=x(t)+$ $y(t)+\frac{p}{\gamma} z(t)$.

Therefore $\frac{d V}{d t}=\frac{d x}{d t}+\frac{d y}{d t}+\frac{p}{\gamma} \frac{d z}{d t}=r x\left(1-\frac{x+y}{k}\right)-\delta y-$ $\frac{p q}{\gamma} z \leq 2 r x-\frac{r}{k} x^{2}-m V=r k-\left(\sqrt{\frac{r}{k}} x-\sqrt{r k}\right)^{2}-m V \leq$ $r k-m V$, where $m=\min (r, \delta, q)$. Hence it follows that $\lim \sup _{t \rightarrow \infty} V(t) \leq \frac{r k}{m}$.

Thus, $z$ is also ultimately bounded by some positive constant and all the solutions of the system $(2 \mathrm{a}-2 \mathrm{c})$, that initiate in $R_{+}^{3}$ are confined in the region

$\Gamma=\left\{(x, y, z) \in R_{+}^{3} / x \leq k, x+y \leq k, x+y+\frac{p}{\gamma} z \leq\right.$ $\left.\frac{r k}{m}\right\}$, which is positively invariant under the flow induced by the system $(2 \mathrm{a}-2 \mathrm{c})$ and the model is well posed.

\subsection{Equilibria}

The system $(2 \mathrm{a}-2 \mathrm{c})$ always has two equilibrium solutions $E_{1}=(0,0,0), E_{2}=(k, 0,0)$. Other steady states are given by

$$
\begin{aligned}
& E_{3}=\left(\frac{(k-\alpha) \gamma-2 q-\gamma \sqrt{(k-\alpha)^{2}-4 k\left(\frac{\beta q}{r \gamma}-\alpha\right)}}{2 \gamma},\right. \\
& \left.\frac{q}{\gamma}, \frac{2(\beta-\delta) k q-r(q+\alpha \gamma)(k+\alpha)-r(q+\alpha \gamma) \sqrt{(k-\alpha)^{2}-4 k\left(\frac{\beta q}{r \gamma}-\alpha\right)}}{2 k p q}\right), \\
& E_{4}=\left(\frac{(k-\alpha) \gamma-2 q+\gamma \sqrt{(k-\alpha)^{2}-4 k\left(\frac{\beta q}{r \gamma}-\alpha\right)}}{2 \gamma}, \frac{q}{\gamma},\right. \\
& \left.\frac{2(\beta-\delta) k q-r(q+\alpha \gamma)(k+\alpha)+r(q+\alpha \gamma) \sqrt{(k-\alpha)^{2}-4 k\left(\frac{\beta q}{r \gamma}-\alpha\right)}}{2 k p q}\right), \\
& E_{5}=\left(\frac{(k+\alpha) \delta r-(\beta-\delta) \delta k-\delta \sqrt{M}}{2 \beta r}, \frac{(\beta-\delta)(k r+\delta k-\beta k)-(\beta+\delta) \alpha r-(\beta-\delta) \sqrt{M}}{2 \beta r}, 0\right), \\
& E_{6}=\left(\frac{(k+\alpha) \delta r-(\beta-\delta) \delta k+\delta \sqrt{M}}{2 \beta r}, \frac{(\beta-\delta)(k r+\delta k-\beta k)-(\beta+\delta) \alpha r+(\beta-\delta) \sqrt{M}}{2 \beta r}, 0\right) \text {, }
\end{aligned}
$$

fected tumor cells, in the presence of the virus specific CTL immune response, is described by the equilibrium state $E_{4}$ provided

$$
\begin{aligned}
& (k-\alpha) \gamma+\gamma \sqrt{(k-\alpha)^{2}-4 k\left(\frac{\beta q}{r \gamma}-\alpha\right)} \geq 2 q \text { and } \\
& 2(\beta-\delta) k q+r(q+\alpha \gamma) \sqrt{(k-\alpha)^{2}-4 k\left(\frac{\beta q}{r \gamma}-\alpha\right)} \geq r(q+
\end{aligned}
$$
$\alpha \gamma)(k+\alpha)$ hold.

If $\beta q>\alpha \gamma r$ and $(k-\alpha)^{2} \geq 4 k\left(\frac{\beta q}{r \gamma}-\alpha\right)$, there is another equilibrium point $E_{3}$, provided the following conditions

$$
\begin{aligned}
& \quad(k-\alpha) \gamma \geq 2 q+\gamma \sqrt{(k-\alpha)^{2}-4 k\left(\frac{\beta q}{r \gamma}-\alpha\right)} \text { and } \\
& 2(\beta-\delta) k q \geq r(q+\alpha \gamma)(k+\alpha)+r(q+\alpha \gamma) \\
& \sqrt{(k-\alpha)^{2}-4 k\left(\frac{\beta q}{r \gamma}-\alpha\right)} \text { hold. }
\end{aligned}
$$

In the absence of CTL response, the coexistence of infected and uninfected tumor cells is described by equilibrium $E_{6}$. The remaining steady state $E_{5}$ is not admissible for the existence of positive equilibrium (The details is described in the Appendix). where $M=\{(k+\alpha) r-(\beta-\delta) k\}^{2}+4 \alpha \beta k r$. Out of these six equilibrium states, $E_{1}$ is the trivial equilibrium state and $E_{2}$ is the equilibrium state corresponding to only healthy tumor cells. $E_{1}$ implies that both the infected and uninfected tumor cells are eliminated in the absence of immune response in due course of time because of the virotherapy. The uninfected steady state $E_{2}$ implies that complete failure of virotherapy which happens when the tumor eventually grows to its carrying capacity. If the condition $\beta q \leq \alpha \gamma r$ is satisfied, $E_{3}$ is not a biologically meaningful equilibrium state. In this case the coexistence of infected and unin-

\subsection{Stability analysis}

To discuss the local stability of equilibrium points, we compute the variational matrix of the system $(2 \mathrm{a}-2 \mathrm{c})$ at any point $(x, y, z)$ and is given by $J=\left(J_{i j}\right)_{3 \times 3}$,

where $J_{11}=\frac{\beta x y}{(x+y+\alpha)^{2}}-\frac{r x}{k}-\frac{\beta y}{x+y+\alpha}+r\left(1-\frac{x+y}{k}\right), J_{12}=$ $\frac{\beta x y}{(x+y+\alpha)^{2}}-\frac{\beta x}{x+y+\alpha}-\frac{r x}{k}, J_{13}=0, J_{21}=\frac{\beta y(y+\alpha)}{(x+y+\alpha)^{2}}, J_{22}=$ $\frac{\beta x}{x+y+\alpha}-p z-\delta-\frac{\beta x y}{(x+y+\alpha)^{2}}, J_{23}=-p y, J_{31}=0, J_{32}=$ $\gamma z, J_{33}=\gamma y-q$. At the steady state $E_{1}$, the eigenvalues of $J$ are $r,-\delta$ and $-q$ and hence $E_{1}$ is always unstable as 
$r$ is positive. Therefore, in the absence of virus and immune response, susceptible cells and hence tumor will grow and the tumor population will move away from the origin.

To see the local stability behaviour of the infection-free equilibrium $E_{2}$, we state and prove the following result.

Theorem 2.3.1 The infection-free equilibrium $E_{2}$ of the system (2a-2c), is locally asymptotically stable for $\rho_{0}<1$ and unstable for $\rho_{0}>1$, where $\rho_{0}$ is the basic reproduction number of the system and is given by $\rho_{0}=\frac{\beta k}{\delta(k+\alpha)}$.

Proof The variational matrix of the system (2a-2c), evaluated at the infection-free steady state $E_{2}$ is given by

$J\left(E_{2}\right)=\left(\begin{array}{ccc}-r & -\frac{\beta k}{(k+\alpha)}-r & 0 \\ 0 & \frac{\beta k}{(k+\alpha)}-\delta & 0 \\ 0 & 0 & -q\end{array}\right)$,

so the eigenvalues are real and are given by $-r,-q$ and $\frac{k \beta}{k+\alpha}-\delta$. Since first two eigenvalues are negative, stability of $E_{2}$ depends on the sign of $\lambda=\frac{k \beta}{k+\alpha}-\delta=\delta\left(\frac{k \beta}{\delta(k+\alpha)}-1\right)=$ $\delta\left(\rho_{0}-1\right)$. When $\lambda<0$, that is, when $\rho_{0}<1$, the steady state $E_{2}$ is asymptotically stable, while it is unstable for $\rho_{0}>1$. As a consequence, $\rho_{0}=1$ is a critical bifurcation value, that is, when the ratio of the cytotoxicity to the replication rate of the viruses equals $\frac{k}{k+\alpha}$, a bifurcation occurs and this can happens only when $\delta<\beta$.

Theorem 2.3.2 The infection-free equilibrium $E_{2}$ of the system (2a-2c), is globally asymptotically stable for $\rho_{0}<1$.

Proof In order to show that $E_{2}$ is globally asymptotically stable, construct a Lyapunov function of the form

$L(x, y, z)=\frac{1}{2}(x(t)-k)^{2}+k y(t)+\frac{p k}{\gamma} z(t)$.

Note that $L(k, 0,0)=0$ and $L(x, y, z)$ is positive for $(x, y, z) \neq(k, 0,0)$ in $R_{+}^{3}$.

$$
\text { Hence } \begin{aligned}
\frac{d L}{d t}= & (x-k) \frac{d x}{d t}+k \frac{d y}{d t}+\frac{p k}{\gamma} \frac{d z}{d t} \\
= & (x-k)\left(r x\left(1-\frac{x+y}{k}\right)-\beta \frac{x y}{x+y+\alpha}\right) \\
& +k\left(\beta \frac{x y}{x+y+\alpha}-\delta y-p y z\right)+\frac{p k}{\gamma}(\gamma y z-q z) \\
= & -r x(k-x)\left(1-\frac{x+y}{k}\right)-\frac{\beta y}{x+y+\alpha}(x-k)^{2} \\
& -k \delta(x+y) \frac{y}{x+y+\alpha}-k\left(1-\frac{\beta k}{\delta \alpha}\right) \frac{\alpha \delta y}{x+y+\alpha} \\
& -\frac{p q k}{\gamma} z .
\end{aligned}
$$

As, in $\Gamma, x \leq k$ and $x+y \leq k$, it is clear that $\frac{d L}{d t}$ is not positive for $\frac{\beta k}{\delta \alpha}<1$, that is, for $\rho_{0}=\frac{\beta k}{\delta(k+\alpha)}<\frac{\beta k}{\delta \alpha}<1$ and for all $(x, y, z)$ in $\Gamma$. Moreover $\frac{d L}{d t}=0$ only when $x=$ $k, y=0, z=0$. Therefore, the largest compact invariant set in $\left\{(x, y, z) \in \Gamma / \frac{d L}{d t}=0\right\}$ is the singleton set $\{(k, 0,0)\}$. From LaSalle's principle of invariance $[25,26]$, we see that $E_{2}=(k, 0,0)$ is globally asymptotically stable for $\rho_{0}<1$.

Regarding the local stability of the interior equilibrium point we have the following result.

Theorem 2.3.3 The interior equilibrium point $E_{4}=(\bar{x}, \bar{y}, \bar{z})$ of the system $(2 \mathrm{a}-2 \mathrm{c})$, is locally asymptotically stable for $r \gamma\left\{k+\alpha+\sqrt{(k+\alpha)^{2}-\frac{4 \beta q}{r \gamma}}\right\}^{2}>4 \beta k q$.

Proof The characteristic equation of the jacobian matrix $J$ at $E_{4}$ can be written as

$\left|J-\lambda I_{3}\right|=\lambda^{3}+a_{1} \lambda^{2}+a_{2} \lambda+a_{3}=0$,

where $a_{1}=\frac{r \bar{x}}{k}, a_{2}=p \gamma \overline{y z}+\frac{r \beta \overline{x y}}{k(\bar{x}+\bar{y}+\alpha)}+\frac{\alpha \beta \overline{x y}}{(\bar{x}+\bar{y}+\alpha)^{3}}, a_{3}=$ $p \gamma \overline{y z}\left(\frac{r \bar{x}}{k}-\frac{\beta \overline{x y}}{(\bar{x}+\bar{y}+\alpha)^{2}}\right)$ and $a_{1} a_{2}-a_{3}=\frac{\delta \overline{x y}}{(\bar{x}+\bar{y}+\alpha)}\left(\frac{r^{2}}{k^{2}} \bar{x}+\right.$

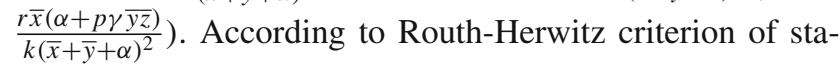
bility, the necessary and sufficient conditions for all the roots of the characteristic equation to have negative real part are $a_{1}>0, a_{3}>0$, and $a_{1} a_{2}-a_{3}>0$. But from the above expressions of $a_{1}$ and $a_{1} a_{2}-a_{3}$, it is clear that $a_{1}>0$ and $a_{1} a_{2}-a_{3}>0$. Thus all the roots of the characteristic equation will have negative real parts, that is the interior equilibrium point $E_{4}=(\bar{x}, \bar{y}, \bar{z})$ is locally asymptotically stable if $a_{3}>0$, that is, if $r \gamma\left\{k+\alpha+\sqrt{(k+\alpha)^{2}-\frac{4 \beta q}{r \gamma}}\right\}^{2}>4 \beta k q$. Similarly it can be shown that another interior equilibrium point $E_{3}$ is locally asymptotically stable if $r \gamma\{k+\alpha-$ $\left.\sqrt{(k+\alpha)^{2}-\frac{4 \beta q}{r \gamma}}\right\}^{2}>4 \beta k q$. Hence the local stability of $E_{3}$ implies the local stability of $E_{4}$.

\subsection{Existence of backward bifurcation}

It is worth noting that, for the restrictions of parameters mentioned in Sect. 2.2 above, two interior equilibria exist which signals the presence of backward bifurcation where a stable interior equilibrium co-exists with a stable infectionfree equilibrium for $\rho_{0}<1$, which has recently received much attention in the epidemiological models [27-30] and has important implications towards the virotherapy strategies, since simply reducing the basic reproduction number less than unity is not enough to control and eliminate the infection/disease. In the presence of backward bifurcation, additional efforts are needed to ensure that the infection is completely eradicated. In the following we will prove that the system $(2 a-2 c)$ exhibits a backward bifurcation when $\rho_{0}=1$. The proof is based on center manifold theory [31], as described in [32] (Theorem 4.1). For this a backward bifurcation parameter, $\beta=\beta^{*}=\frac{\delta(k+\alpha)}{k}$, is taken, which is equivalent to $\rho_{0}=1$. Now the jacobian $J_{\beta^{*}}\left(E_{2}\right)$ of the system 
(2a-2c) evaluated at infection-free equilibrium $E_{2}$ with $\beta=$ $\beta^{*}$ becomes

$J_{\beta^{*}}\left(E_{2}\right)=\left(\begin{array}{ccc}-r & -\delta-r & 0 \\ 0 & 0 & 0 \\ 0 & 0 & -q\end{array}\right)$.

Clearly eigenvalues of this matrix are $\lambda_{1}=-r, \lambda_{2}=$ $0, \lambda_{3}=-q$. Thus $J_{\beta^{*}}\left(E_{2}\right)$ has a simple eigenvalue and other two are real and negative. Let $w$ and $v$ be its right and left eigenvectors respectively corresponding to the eigenvalue $\lambda_{2}=0$, then $w=\left(\frac{\delta+r}{r},-1,0\right)^{t}$ and $v=(0,-1,0)$ satisfying $w \cdot v=1$. The coefficients $a$ and $b$, defined in theorem 4.1 in the paper of Castillo-Chavez and Song [32]

$a=\sum_{k, j, i=1}^{3} v_{k} w_{i} w_{j} \frac{\partial^{2} f_{k}}{\partial x_{i} \partial x_{j}}\left(E_{2}, \beta^{*}\right)$,

$b=\sum_{k, i=1}^{3} v_{k} w_{i} \frac{\partial^{2} f_{k}}{\partial x_{i} \partial \beta}\left(E_{2}, \beta^{*}\right)$,

may be now explicitly calculated as follows. Considering only the nonzero components of the left eigenvector $v$ and taking into account of system $(2 \mathrm{a}-2 \mathrm{c}), a$ and $b$ reduced to
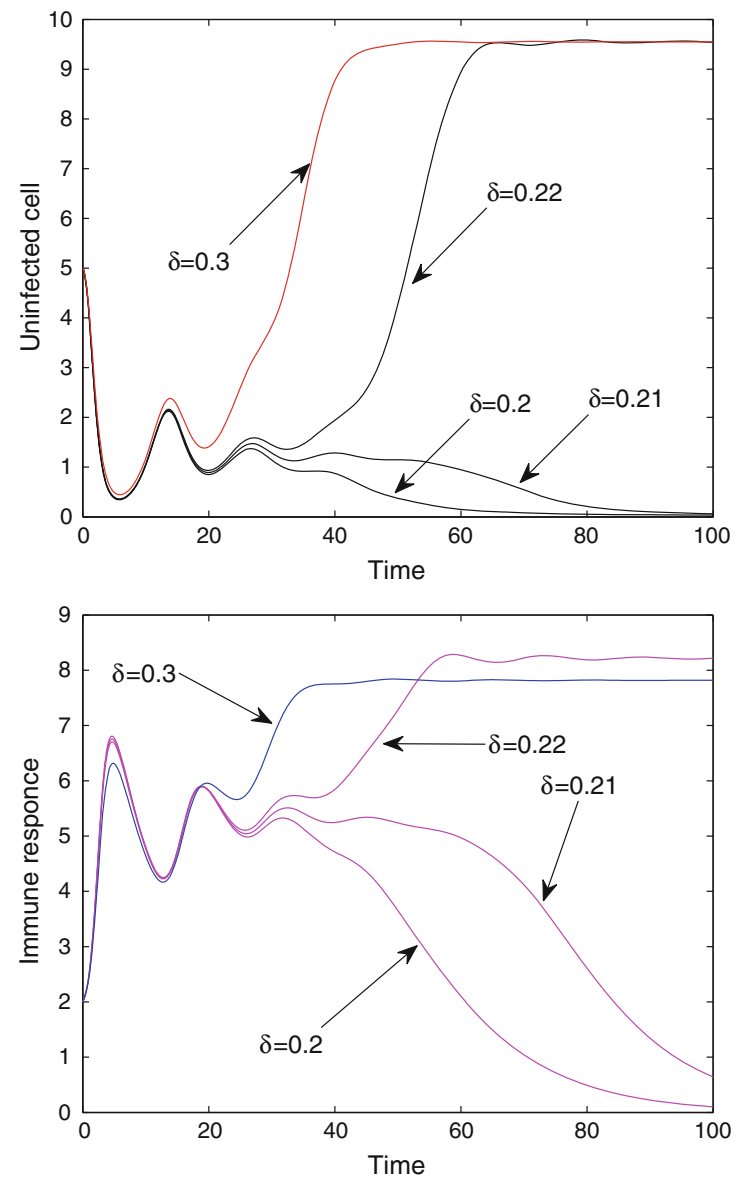

$$
\begin{aligned}
a= & v_{2} w_{1}^{2} \frac{\partial^{2} f_{2}}{\partial x^{2}}\left(E_{2}, \beta^{*}\right)+2 v_{2} w_{1} w_{2} \frac{\partial^{2} f_{2}}{\partial x \partial y}\left(E_{2}, \beta^{*}\right) \\
& +2 v_{2} w_{1} w_{3} \frac{\partial^{2} f_{2}}{\partial x \partial z}\left(E_{2}, \beta^{*}\right)+2 v_{2} w_{2} w_{3} \frac{\partial^{2} f_{2}}{\partial y \partial z}\left(E_{2}, \beta^{*}\right) \\
& +v_{2} w_{2}^{2} \frac{\partial^{2} f_{2}}{\partial y^{2}}\left(E_{2}, \beta^{*}\right)+v_{2} w_{3}^{2} \frac{\partial^{2} f_{2}}{\partial z^{2}}\left(E_{2}, \beta^{*}\right), \\
b= & v_{2} w_{1} \frac{\partial^{2} f_{2}}{\partial x \partial \beta}\left(E_{2}, \beta^{*}\right)+v_{2} w_{2} \frac{\partial^{2} f_{2}}{\partial y \partial \beta}\left(E_{2}, \beta^{*}\right) \\
& +v_{2} w_{3} \frac{\partial^{2} f_{2}}{\partial z \partial \beta}\left(E_{2}, \beta^{*}\right) .
\end{aligned}
$$

As in our system $x_{1}=x, x_{2}=y, x_{3}=z$ and

$$
\begin{aligned}
& f_{1}=r x\left(1-\frac{x+y}{k}\right)-\beta \frac{x y}{x+y+\alpha}, \\
& f_{2}=\beta \frac{x y}{x+y+\alpha}-\delta y-p y z \\
& f_{3}=\gamma y z-q z .
\end{aligned}
$$

Second order Partial derivatives contained in the expressions of $a$ and $b$ are computed as
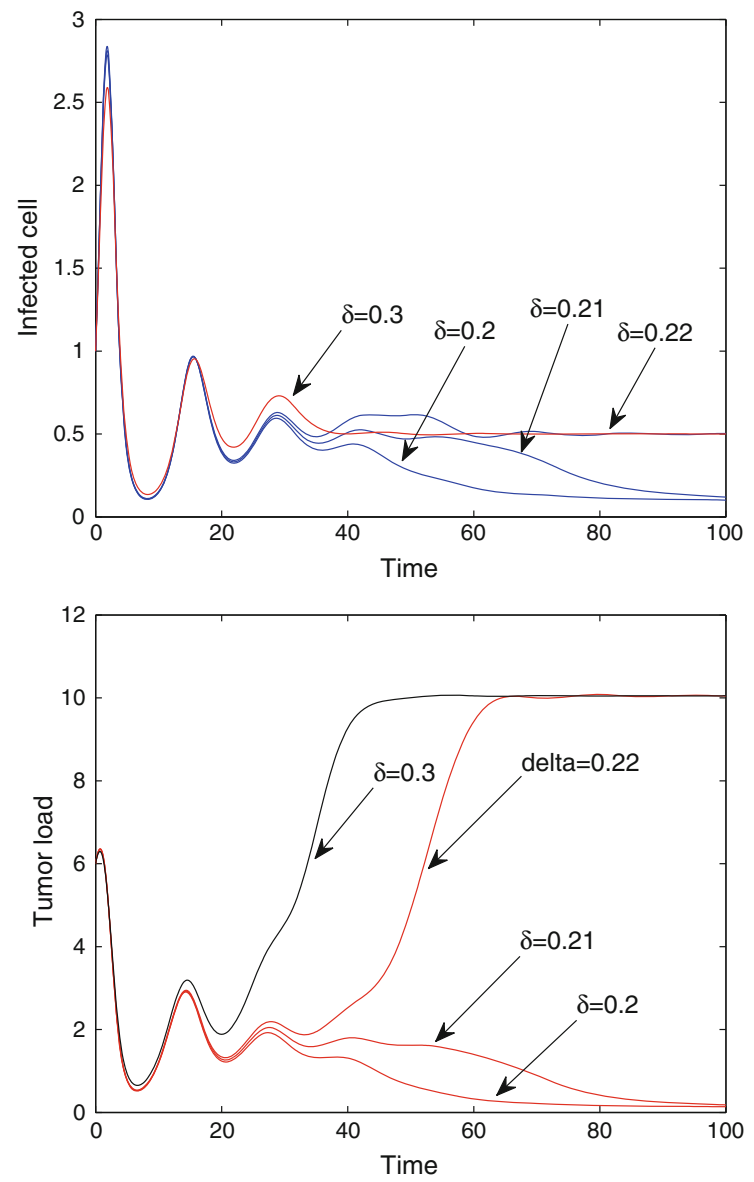

Fig. 1 For four values of viral cytotoxicity, $\delta$, uninfected cells, infected cells, immune responses and overall tumor sizes are shown starting with the initial conditions $x(0)=5, y(0)=1$ and $z(0)=2$. Other parameters are fixed at $r=0.6, k=12, \alpha=0.2, \beta=2, \gamma=0.2, p=0.2$ and $q=0.1$ 


$$
\begin{aligned}
\frac{\partial^{2} f_{2}}{\partial x^{2}}\left(E_{2}, \beta^{*}\right) & =0, \frac{\partial^{2} f_{2}}{\partial x \partial y}\left(E_{2}, \beta^{*}\right) \\
& =\frac{\alpha \delta}{k(k+\alpha)}, \frac{\partial^{2} f_{2}}{\partial x \partial z}\left(E_{2}, \beta^{*}\right)=0, \\
\frac{\partial^{2} f_{2}}{\partial y^{2}}\left(E_{2}, \beta^{*}\right) & =-\frac{2 \delta}{k+\alpha}, \frac{\partial^{2} f_{2}}{\partial y \partial z}\left(E_{2}, \beta^{*}\right) \\
& =-p, \frac{\partial^{2} f_{2}}{\partial z^{2}}\left(E_{2}, \beta^{*}\right)=0 .
\end{aligned}
$$

Therefore $a=\frac{2 \delta(\delta+2 r)}{r(k+\alpha)}$ and $b=\frac{k}{k+\alpha}$. Hence both $a>0$ and $b>0$, which shows, from the theorem 4.1 of [32], that the system $(2 \mathrm{a}-2 \mathrm{c})$ undergoes a backward bifurcation at $\rho_{0}=1$.

\section{Numerical simulation}

In this section, we study numerically the basic dynamics between a growing tumor cell, a replicating oncolytic virus selective for the tumor cells and a specific cytotoxic T lymphocyte response by observing the effect of variations in the model parameters. In order to study the effects of parameter
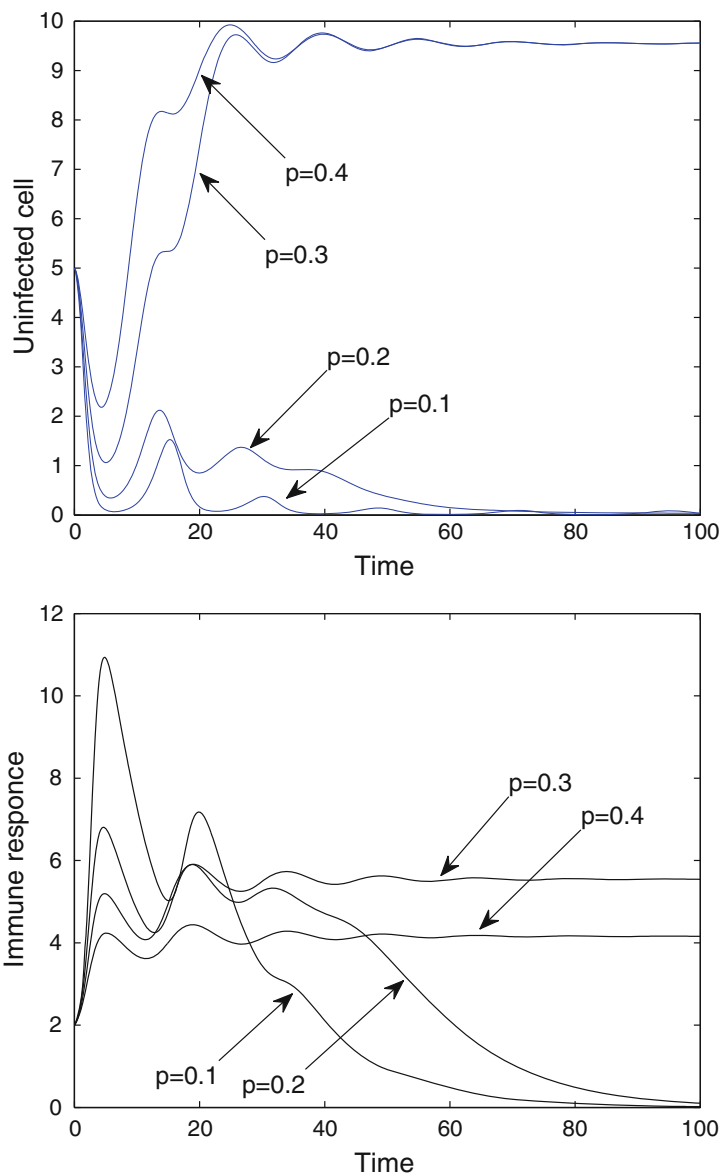

Fig. 2 For four different values of $p$, the strength of the lytic component, the densities of uninfected cells, infected cells, CTL responses and tumor sizes are shown. Other parameters are fixed at $r=0.6, k=$ changes in the model $(2 \mathrm{a}-2 \mathrm{c})$, we run simulations using the standard matlab differential integrator.

Toxicity is a primary concern with any experimental therapeutic agent and virotherapy agents are no different. The toxicity will depend on the virus strain used, the specific genetic changes made, and the dose of administration. To consider the effect of cytotoxicity of the virus on overall tumor load, we use a set of parameter values $r=0.6$ time $^{-1}, k=$ 12 cells $/ \mathrm{mm}^{3}, \alpha=0.2$ cells $/ \mathrm{mm}^{3}, \beta=2$ time $^{-1}, \gamma=$ $0.2 \mathrm{~mm}^{3} /$ cells/time, $p=0.2 \mathrm{~mm}^{3} /$ cells/time and $q=0.1$ time $^{-1}$. In Fig. 1, uninfected cells, infected cells, immune response and overall tumor sizes are presented graphically for four different values of cytotoxicity $\delta$, say, for $\delta=$ $0.2,0.21,0.22$ and 0.3 time $^{-1}$. For the above set of fixed values of the parameters and for low level of cytotoxicity, here $\delta<0.22$, tumor size reduces and we obtain tumor eradication. An increase in the viral cytotoxicity above 0.22 increases tumor size, that is, for more cytotoxic viruses with $\delta \geq 0.22$ tumor persistence is observed and hence the therapy fails. At the same time, virus specific CTL cells persist at a higher level. The explanation of this phenomena is the
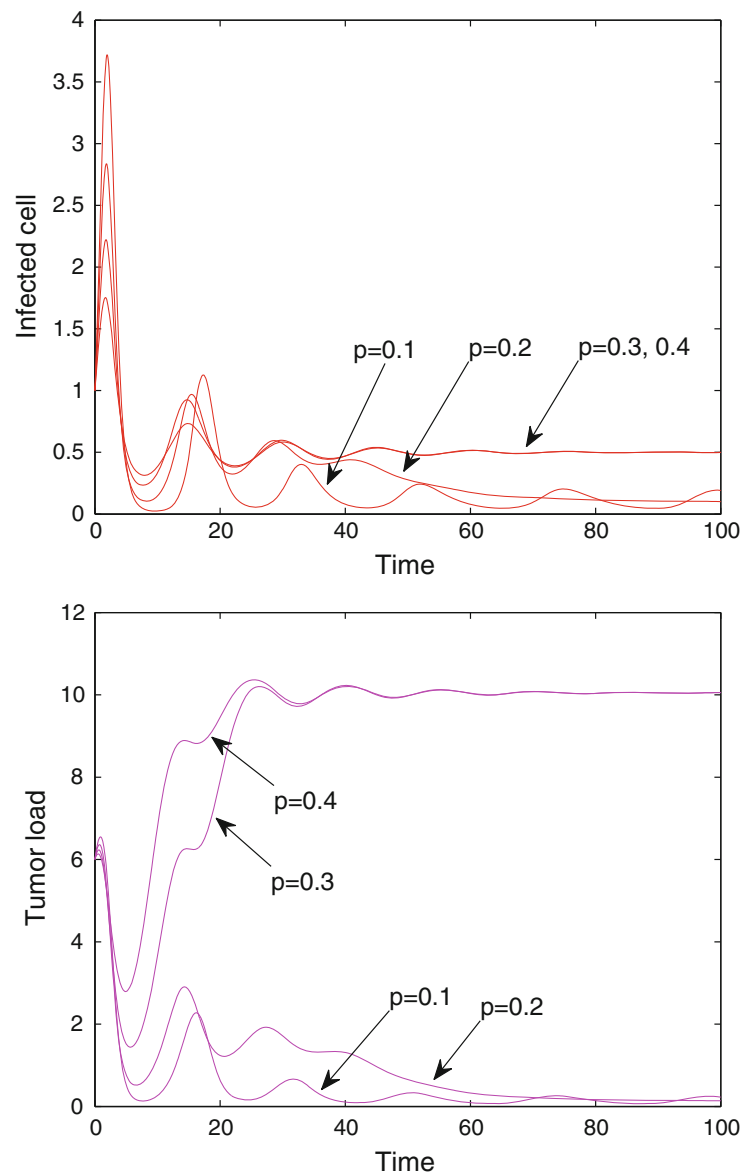

$12, \alpha=0.2, \beta=2, \gamma=0.2, \delta=0.2$, and $q=0.1$. The initial populations are $x(0)=5, y(0)=1$ and $z(0)=2$ 

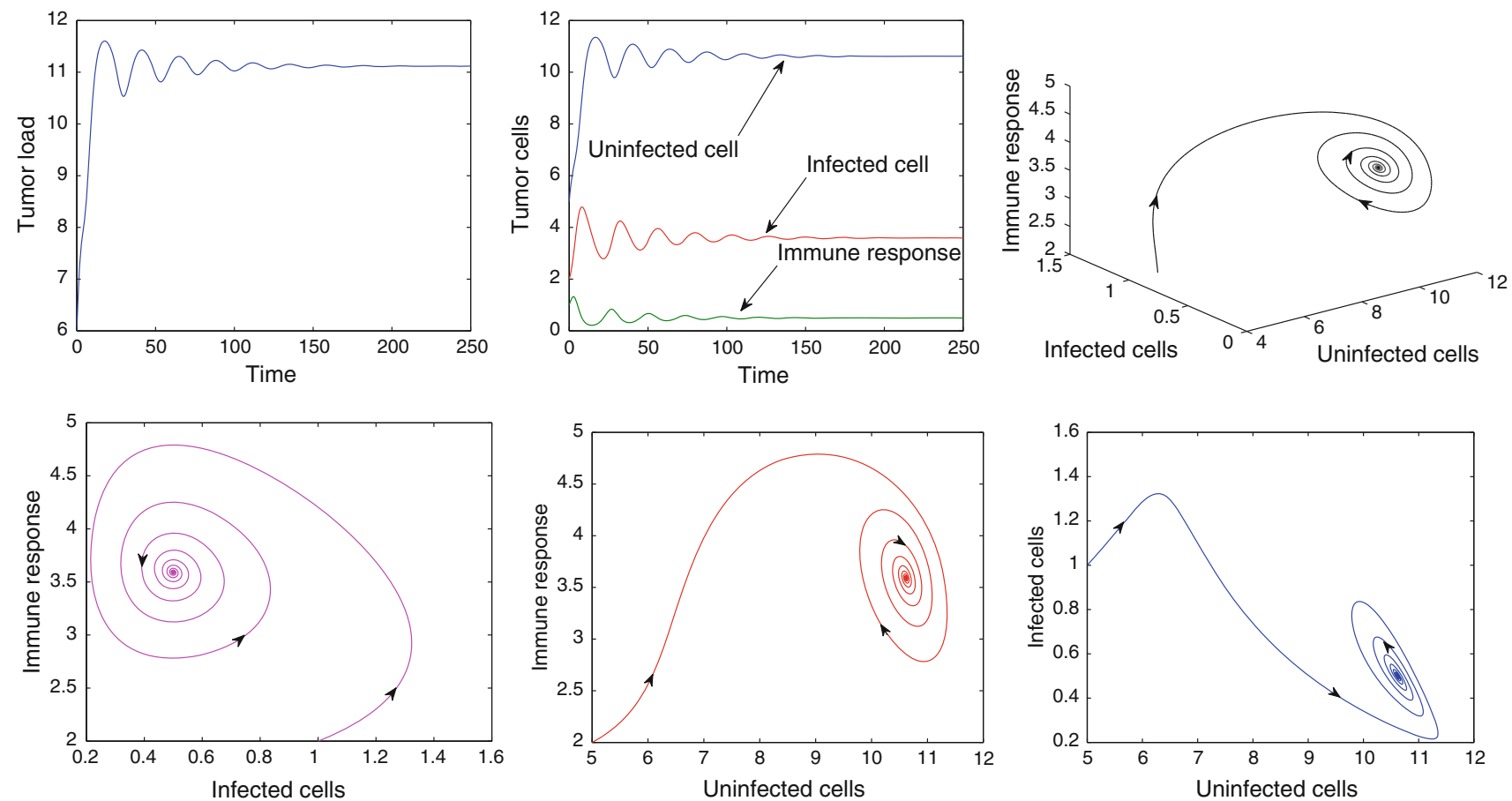

Fig. 3 Time trajectories, three dimensional phase portrait, $x-y, y-z$ and $x-z$ sub phase spaces depicting stable dynamics of the system (2) for the parameter values $r=0.6, k=12, \alpha=0.2, \beta=1, \gamma=0.2, \delta=0.22, p=0.2$ and $q=0.1$, starting from the initial conditions $x(0)=5, y(0)=1$ and $z(0)=2$

following. The increased rates of tumor cell killing reduce infected tumor cells before the virus has a chance to properly spread. This opens the scope of increase in the pool of uninfected tumor cells and, therefore, the tumor size increases. The virotherapy thus becomes ineffective.

Figure 2 shows similar features on the effect of the strength of lytic component $p$. In the presence of CTL, infected cells, uninfected cells and tumor sizes are shown for four different values of $p$, say, $p=0.1,0.2,0.3$ and 0.4 . For $p \leq 0.2$, overall tumor size reduces and results in tumor eradication. For $p>0.2$, tumor persists which is detrimental to the patient. We also see that immune response remains at a higher level for this range of $p$. There is a natural explanation of it which is that the CTL response kills the virus faster than the rate at which it can spread.

Figure 3 illustrates the approach of the trajectories to the steady state $E_{4}$, which is asymptotically stable for the parameter values as mentioned in the caption of the figure. For this set of numerical values of the parameters, $E_{3}$ is not feasible. The positive equilibrium, in the presence of immune response, is $E_{4}=(10.6163,0.5000,3.5907)$ and in fact, the eigenvalues of the variational matrix of the system $(2 a-2 c)$ at $E_{4}$ are $-0.4828,-0.240+0.2687 \mathrm{i}$ and $-0.240-0.2687 \mathrm{i}$. The real part of the eigenvalues are all negative, which results in an asymptotically stable state.

In Figs. 4 and 5, tumor load, densities of uninfected cell, infected cell and CTL response versus time are plotted for the parameter values as mentioned in the caption of the figures to observe the effects of immune responsiveness $\gamma$ on tumor size. Figure 4 shows a simulation of therapy where we observe that for low level of CTL responsiveness $\gamma(\leq 0.1)$ tumor size reaches very low level. In this range of $\gamma$ it is possible to eradicate the tumor and hence we have a successful therapy of cancer. For a higher strength of CTL response $\gamma(>0.1)$ tumor size increases and leads to the persistence of tumor. In Fig. 4 tumor size is also shown for $\gamma=0.2$, which shows tumor persistence. For more strong CTL responsiveness $\gamma$, say for $\gamma=2$, tumor size tends to its carrying capacity as shown in Fig. 5. Our numerical experience suggests that higher CTL response can result in failure of therapy. This is due to the fact that the presence of strong CTL response increases the death rate of infected cells and hence viruses destroy at a higher rate and consequently the spread of the virus from cell-to-cell is inhibited. As $\gamma$ increases healthy cells increase and infected cells decrease. We see that a weak CTL response controls the cancer to lower level. Regarding the virotherapy, the mathematical model suggests that the therapy works better in the situation when CTL response lies below certain level. In most virus dynamics studies, viral replication rate is also an essential parameter which plays an important role in defining the oncolytic virus treatment outcome. Figures 6 and 7 show variation of tumor sizes with time for different transmission rates of virus infection. 

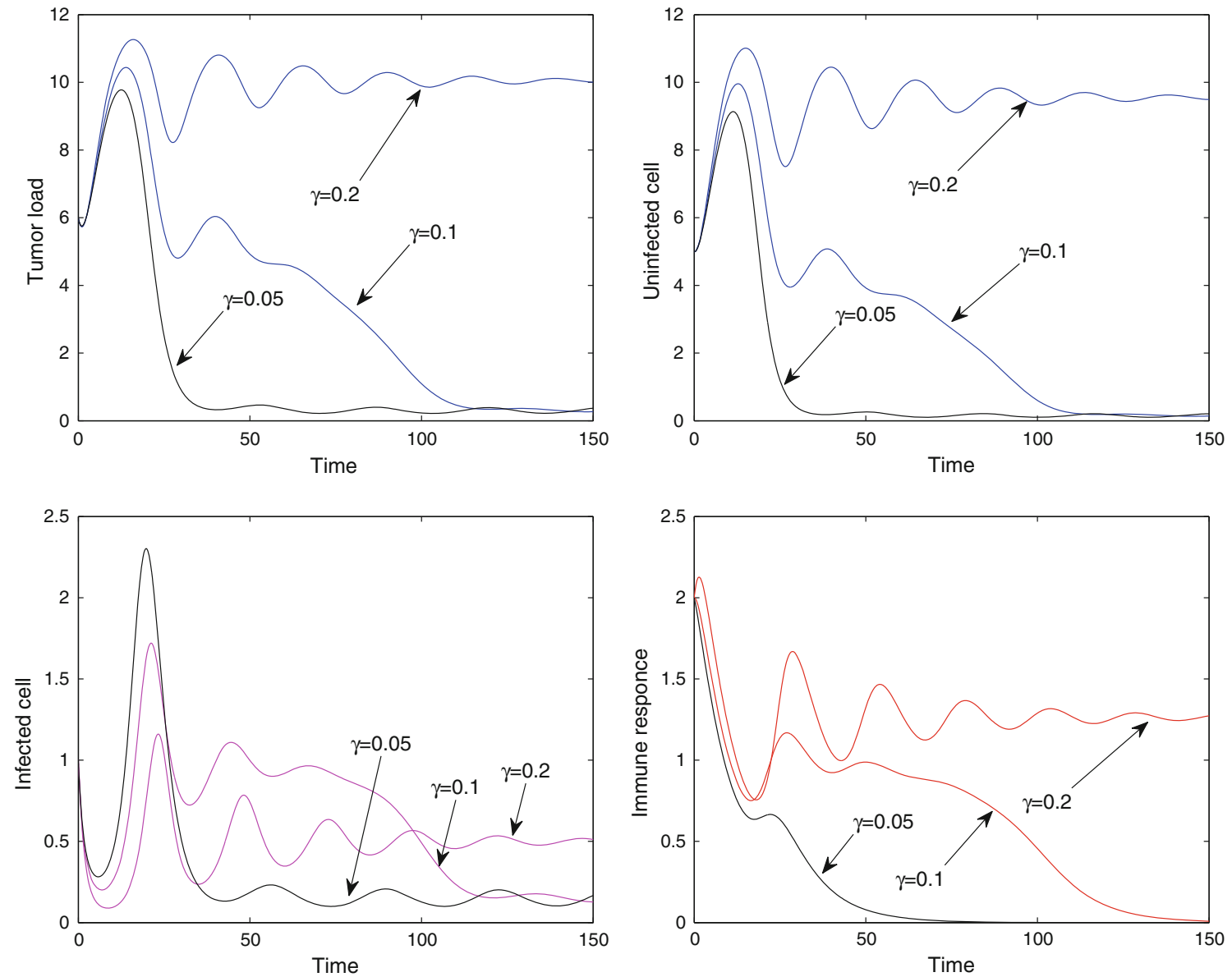

Fig. 4 For three values of immune responsiveness $\gamma$, tumor loads, densities of uninfected cells, infected cells and CTL responses are shown. Other parameters are fixed at $r=0.3, k=12, \alpha=0.2, \beta=1, \delta=0.3, p=0.5$ and $q=0.1$. The initial conditions are $x(0)=5, y(0)=1$ and $z(0)=2$
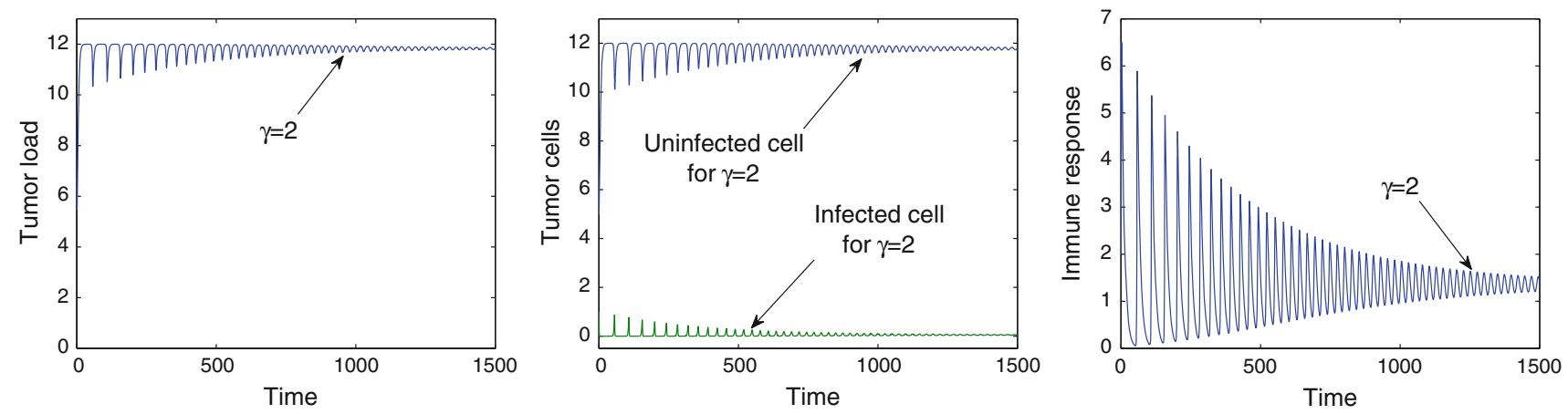

Fig. 5 For more higher value of immune responsiveness $\gamma$, tumor load, densities of uninfected cells, infected cells and immune response are shown. Other parameters are fixed at $r=0.3, k=12, \alpha=0.2, \beta=1, \delta=0.3, p=0.5$ and $q=0.1$. The initial conditions are $x(0)=5, y(0)=1$ and $z(0)=2$

It is observed from our model that increasing the rate of virus replication will improve the chances of virotherapy success when the virus spreading term belongs to the fast spreading class [5]. Virus load decreases with the increase in $\beta$. In Fig. 6 the densities of uninfected cells, infected cells and tumor size are drawn for $\beta=2$.
Other parameters are chosen as follows $r=0.3, k=$ $12, \alpha=3, \gamma=0.2, \delta=0.3, p=0.5$ and $q=0.1$. The tumor cell population converges to the steady state $E_{4}(8.0311,0.50000,2.1859)$ which is asymptotically stable as it is evident from the phase plane of uninfected and infected cells. 

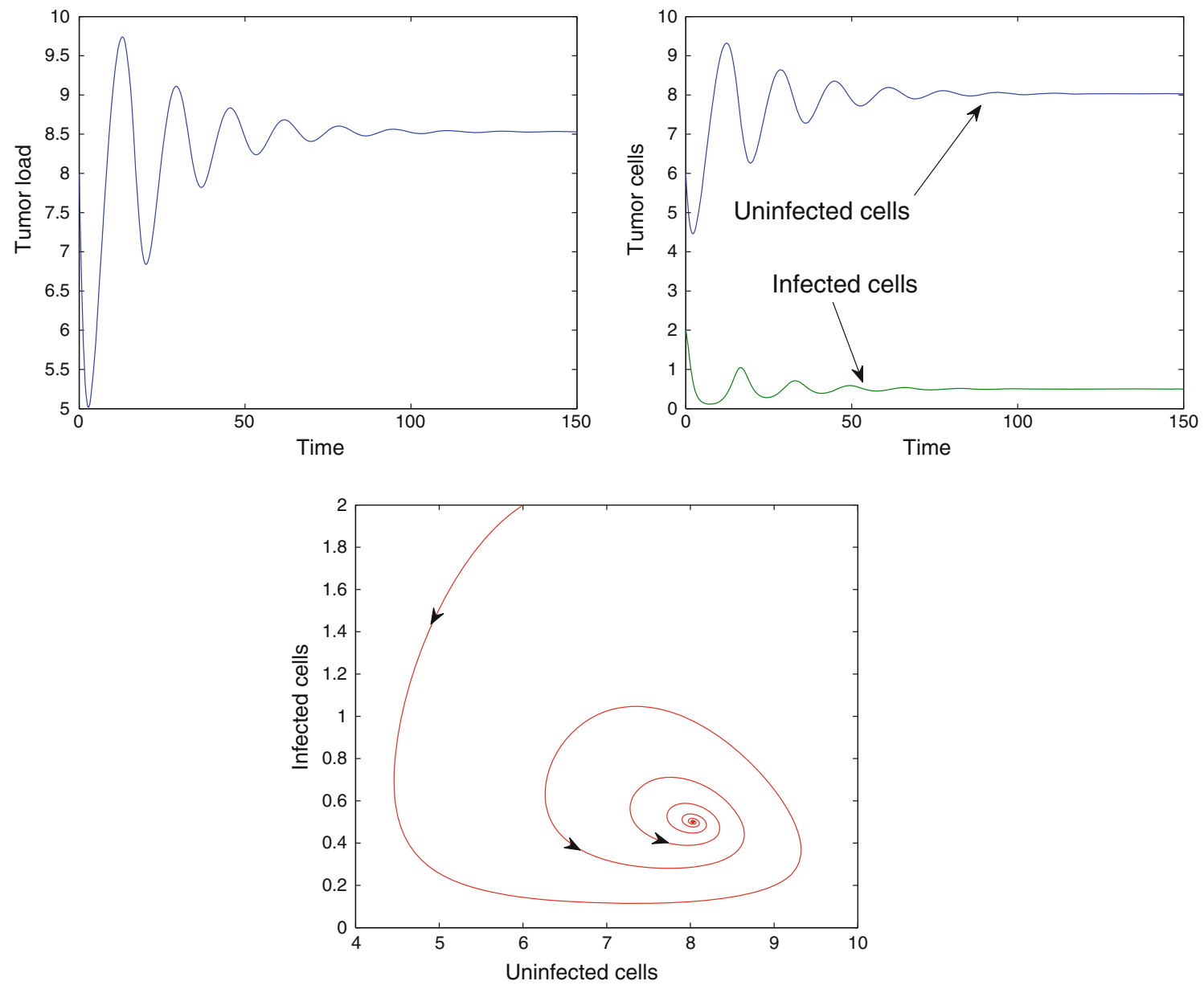

Fig. 6 Tumor load, densities of uninfected cells, infected cells and $x-y$ phase plane are shown for the parameters values $r=0.3, k=12$, $\alpha=3, \beta=2, \gamma=0.2, \delta=0.3, p=0.5$ and $q=0.1$. The initial conditions were chosen as $x(0)=6, y(0)=2$ and $z(0)=2$
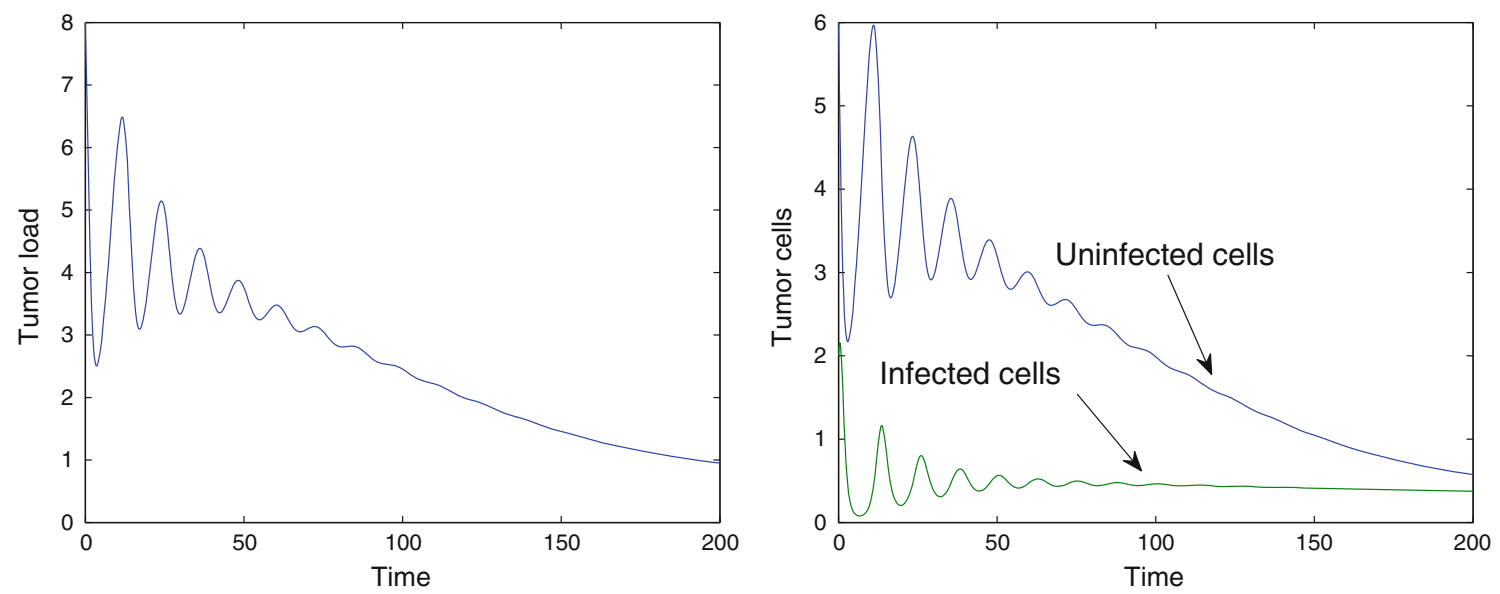

Fig. 7 Total tumor cells and the densities of uninfected cells, infected cells are shown separately for infectivity rate $\beta=3$. Other parameters are $r=0.3, k=12, \alpha=3, \gamma=0.2, \delta=0.3, p=0.5$ and $q=0.1$ and the initial conditions are $x(0)=6, y(0)=2$ and $z(0)=2$

In plotting of the tumor cells in Fig. 7, we take a higher replication rate $\beta=3$ and see that virus therapy is beneficial to the patient as the tumor size reduces to very low level. For further increase in infectivity we obtain damped oscilla- tions leading the system towards an equilibrium. The level of virus load at this equilibrium shows how well the infection is controlled. For a higher level of CTL responsiveness, higher infectivity rate is required as it is expected. In Fig. 8, 

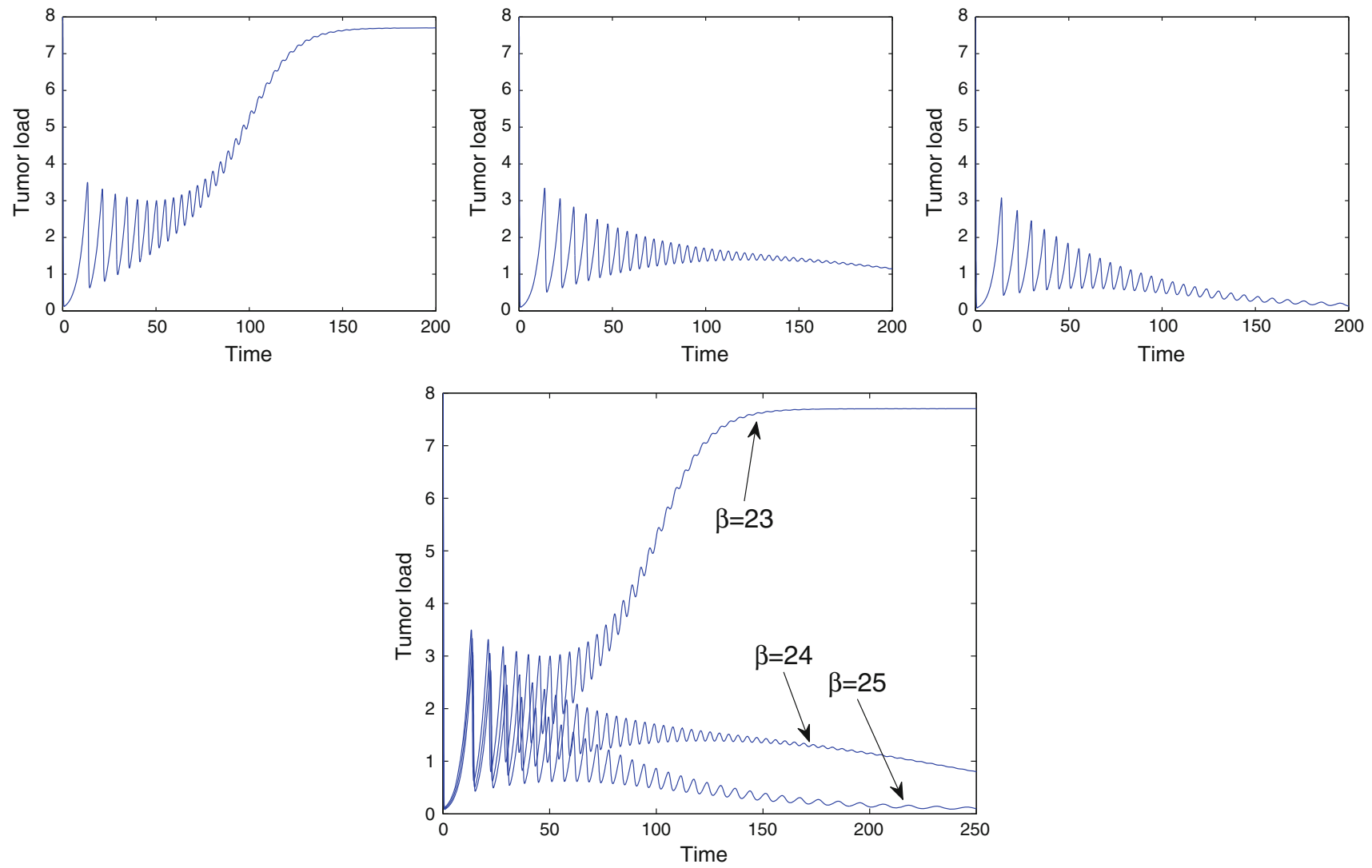

Fig. 8 For three values of infectivity rate $\beta$, tumor loads are shown. Other parameters are fixed at $r=0.3, k=12, \alpha=3, \gamma=2, \delta=0.3, p=0.5$, and $q=0.1$. The initial conditions are $x(0)=6, y(0)=2$ and $z(0)=2$
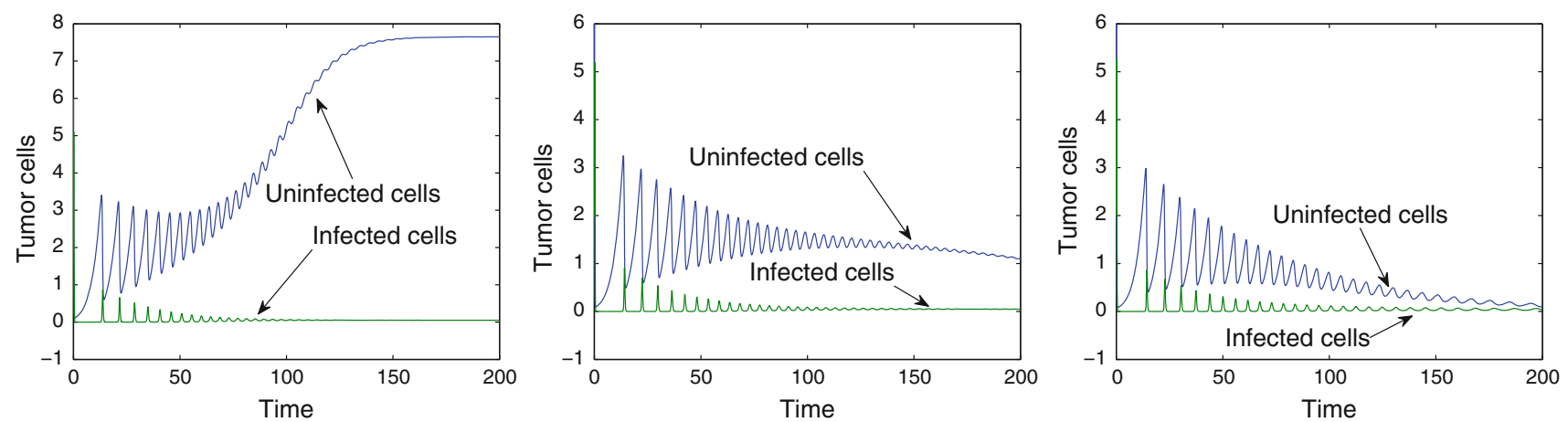

Fig. 9 Uninfected cells, infected cells are shown separately for the parameter values as in Fig. 8

we take a higher level of CTL responsiveness $\gamma$, say, $\gamma=2$. For this value of $\gamma$, tumor sizes are shown for three different higher values of infectivity rate $\beta$, say for $\beta=23,24$ and 25 . For $\beta \leq 23$ tumor size remains above certain level and consequently virotherapy fails. When $\beta>23$ tumor size eventually reaches very low level after damped oscillations. Figure 9 displays tumor cell populations, uninfected and infected, depicting the control of tumor cells. Specific feature of the dynamics is strong oscillations which is supposed to be clinically reflected during treatment.

\section{Conclusion}

In this paper we make a numerical study of the viability of a virotherapy of cancer in the presence of an immune system. The final finding of our study is that the therapy can be effective, even it is possible to drive the population of the tumor cell to zero, that is, a complete cure of the disease, provided that certain parameter values can be adjusted. These parameters either pertain to the system itself, such as the level of immune response, or related to the administration 
of the therapy, such as the infectivity of the oncolytic virus. The dynamics of the population of two types of tumor cells, uninfected and infected, is studied in the presence of the immune response of the body. How the parameters indicated for the control of tumor cell population can be adjusted is a question to be addressed by the biologist.

\section{Appendix}

For the positive equilibrium $(x, y, z)$, we have

$$
\begin{aligned}
r\left(1-\frac{x+y}{k}\right)-\beta \frac{y}{x+y+\alpha} & =0, \\
\beta \frac{x}{x+y+\alpha}-\delta-p z & =0, \\
\gamma y-q & =0 .
\end{aligned}
$$

Substituting $x$ and $y$ from (3b) and (3c) respectively, in equation (3a) we obtain a quadratic equation

$F(z)=A z^{2}+B z+C=0$ in $z$

where the coefficients $A, B$ and $C$ are given by

$$
\begin{aligned}
A= & p^{2}\left(\frac{q}{\gamma}+\alpha\right)^{2}-p^{2}\left(\frac{q}{\gamma}+\alpha\right)\left(\frac{q}{\gamma}+\alpha+\frac{q}{\gamma}-k\right) \\
& +p^{2}\left\{\frac{\beta k q}{r \gamma}-\left(k-\frac{q}{\gamma}\right)\left(\frac{q}{\gamma}+\alpha\right)\right\}=p^{2} \frac{\beta k q}{r \gamma}, \\
B= & 2 p \delta\left(\frac{q}{\gamma}+\alpha\right)^{2}+\left(\frac{q}{\gamma}+\alpha\right)\left(\frac{q}{\gamma}+\alpha+\frac{q}{\gamma}-k\right) \\
& (p \beta-2 p \delta)-2 p(\beta-\delta)\left\{\frac{\beta k q}{r \gamma}-\left(k-\frac{q}{\gamma}\right)\left(\frac{q}{\gamma}+\alpha\right)\right\},
\end{aligned}
$$

and

$$
\begin{aligned}
C= & \delta^{2}\left(\frac{q}{\gamma}+\alpha\right)^{2}+\left(\frac{q}{\gamma}+\alpha\right)\left(\frac{q}{\gamma}+\alpha+\frac{q}{\gamma}-k\right) \beta \delta \\
& +(\beta-\delta)^{2}\left\{\frac{\beta k q}{r \gamma}-\left(k-\frac{q}{\gamma}\right)\left(\frac{q}{\gamma}+\alpha\right)\right\} .
\end{aligned}
$$

The quadratic equation $F(z)=0$, can be analyzed for the possibility of multiple non-negative equilibria under certain conditions among the model parameters. Since the coefficient $A$ ia always positive, the existence of the positive solutions of equation (4) will depend on the signs of $B$ and $C$. There is a unique positive root of the equation (4) if $C<0$ and whatever be the sign of $B$ and thus in this case we have an unique interior equilibrium. If $C=0$, then there is an unique positive solution of (4) if and only if $B<0$. If $C>0$ and either $B \geq 0$ or $B^{2}-4 A C<0$, there are no positive solution. If $C>0$, and $B<0, B^{2}-4 A C>0$, The equation (4) has two positive solutions corresponding to two interior equilibria of the system (2a, 2c). One can verify that $C>0$ and $B<0$ can occur for some parameter values, and thus we have an indication of the possibility of a backward bifurcation. To check for this, we set $B^{2}-4 A C=0$ and solve for the critical value $\rho_{0}^{*}$, say, of $\rho_{0}$. Hence $\rho_{0}^{*}<\rho_{0}$ is equivalent to $B^{2}-4 A C>0$ and, thus, backward bifurcation would occur for values of $\rho_{0}$ such that $\rho_{0}^{*}<\rho_{0}<1$. We do not present here the explicit expression for $\rho_{0}^{*}$ because it is rather cumbersome.

\section{References}

1. Aghi M, Martuza RL (2005) Oncolytic viral therapies-the clinical experience. Oncogene 24:7802-7816

2. Bajzer Z, Carr T, Josic K, Russell SJ, Dingli D (2008) Modeling of cancer virotherapy with recombinant measles viruses. J Theor Biol 252:109-122

3. Crompton AM, Kirn DH (2007) From ONYX-015 to armed vaccinia viruses: the education and evolution of oncolytic virus development. Curr Cancer Drug Targets 7:133-139

4. Davis JJ, Fang B (2005) Oncolytic virotherapy for cancer treatment: challenges and solutions. J Gene Med 7:1380-1389

5. Komarova NL, Wodarz W (2010) ODE models for oncolytic virus dynamics. J Theor Biol 263:530-543

6. Kasuya H, Takeda S, Nomoto S, Nakao A (2005) The potential of oncolytic virus therapy for pancreatic cancer. Cancer Gene Ther $12: 725-736$

7. Kaplan JM (2005) Adenovirus-based cancer gene therapy. Curr Gene Ther 5:595-605

8. Kirn DH, McCormick F (1996) Replicating viruses as selective cancer therapeutics. Mol Med Today 2:519-537

9. Lorence RM, Pecora AL, Major PP, Hotte SJ, Laurie SA, Roberts MS, Groene WS, Bamat MK (2003) Overview of phase I studies of intravenous administration of PV701, an oncolytic virus. Curr Opin Mol Ther 5:618-624

10. McCormick F (2003) Cancer-specific viruses and the development of ONXY-015. Cancer Biol Ther 2:157-160

11. McCormick F (2005) Future prospects for oncolytic therapy. Oncogene 24:7817-7819

12. Nowak MA, May RM (2000) Virus dynamics: mathematical principles of immunology and virology. Oxford University Press, Oxford

13. Parato KA, Senger D, Forsyth PA, Bell JC (2005) Recent progress in the battle between oncolytic viruses and tumors. Nat Rev Cancer 5:965-976

14. Wodarz D (2001) Viruses as antitumor weapons: defining conditions for tumor remission. Cancer Res 61:3501-3507

15. Wodarz D (2003) Gene therapy for killing p53-negative cancer cells: use of replicating versus non replicating agents. Hum Gene Ther 14:153-159

16. Wodarz D, Komarova NL (2005) Computational biology of cancer. Lecture notes and mathematical modeling. World Scientific, Singapore

17. Wodarz D, Komarova NL (2009) Towards predictive computational models of oncolytic virus therapy: basis for experimental validation and model selection. PLoS ONE 4:e4271

18. Novozhilov AS, Berezovskaya FS, Koonin EV, Karev GP (2006) Mathematical modeling of tumor therapy with oncolytic viruses: regimes with complete tumor elimination within the framework of deterministic models. Biol Direct I:6

19. Burnet FM (1957) Cancer-a biological approach. BMJ 1:841-847

20. De Boer RJ, Perelson AS (1995) Towards a general function describing T cell proliferation. J Theor Biol 175:567-576

21. De Boer RJ, Perelson AS (1998) Target cell limited and immune controls of HIV infection: a comparison. J Theor Biol 190:201-214 
22. Xie Q, Huang D, Zhang S, Cao J (2010) Analysis of a viral infection model with delayed immune response. Appl Math Model 34:23882395

23. Wodarz D, Chrristensen JP, Thomsen AR (2002) The importance of lytic and nonlytic immune responses in viral infections. Trends Immunol 23:194-200

24. Wang K, Wang W, Liu X (2006) Viral infection model with periodic lytic immune response. Chaos Solitons Fract 28:90-99

25. Hale JK, Verduyn Lunel S (1993) Introduction to functional differential equations. Springer, New York

26. LaSalle JP (1976) The stability of dynamical systems, regional conference series in applied mathematics. SIAM, Philadelphia

27. Gomez-Acevedo H, Li MY (2005) Backward bifurcation in a model for HTLV-I infection of $C D 4^{+}$T cells. Bull Math Biol 67:101-114
28. Brauer F (2004) Backward bifurcations in simple vaccination models. J Math Anal Appl 298:418-431

29. Arino J, McCluskey CC, Van den Driessche P (2003) Global results for an epidemic model with vaccination that exhibits backward bifurcation. SIAM J Appl Math 64:260-276

30. Dushoff J, Huang W, Castillo-Chavez C (1998) Backwards bifurcations and catastrophe in simple models of fatal diseases. J Math Biol 36:227-248

31. Carr J (1981) Applications of centre manifold theory. Springer, New York

32. Castillo-Chavez C, Song B (2004) Dynamical models of tuberculosis and their applications. Math Biosci Eng 1:361-404 